\title{
O bullying na primeira infância: revisão integrativa da literatura
}

\author{
Bullying in early childhood: integrative literature review
}

Bullying en la primera infancia: revisión integradora de la literatura

Aline Oliveira Paulino dos Santos

Graduanda na Universidade de São Paulo, São Paulo, SP, Brasil

E-mail: alineoliveirap@usp.br ORCID: https://orcid.org/0000-0002-1575-4062

Jennifer Stephanie Marques Hilario

Mestre em Ciências pela Universidade de São Paulo, São Paulo, SP, Brasil

E-mail: jsmhilario@outlook.com ORCID: https://orcid.org/0000-0001-5541-6546

Rosane Meire Munhak Silva

Professora adjunta na Universidade Estadual do Oeste do Paraná, Cascavel, PR, Brasil

E-mail: zanem2010@hotmail.com ORCID: https://orcid.org/0000-0003-3355-0132

Marta Angélica lossi Silva

Professora associada na Universidade de São Paulo, São Paulo, SP, Brasil

E-mail: maiossi@eerp.usp.br ORCID: https://orcid.org/0000-0002-9967-8158

Débora Falleiros de Mello

Professora titular na Universidade de São Paulo, São Paulo, SP, Brasil

E-mail: defmello@eerp.usp.br ORCID: https://orcid.org/0000-0001-5359-9780

Recebido em 19 de outubro de 2020

Aprovado em 04 de janeiro de 2021

Publicado em 20 de janeiro de 2021

\section{RESUMO}

Identificar evidências científicas sobre as repercussões do bullying na primeira infância. Revisão integrativa da literatura que analisou 13 artigos, das bases de dados PUBMED/MEDLINE, LILACS, CINAHL, Web of Science e PSYCOINFO a partir das palavras-chave criança, bullying e desenvolvimento infantil, no período 2009 a 2019, nos idiomas português e inglês. As informações foram agrupadas nas categorias temáticas: Bullying na primeira infância: aspectos relacionais e contextuais; Cuidados parentais adversos e interações agressivas; Bullying em crianças em circunstâncias específicas. As questões que envolvem o bullying na primeira infância podem ter repercussões ligadas ao processo de estresse tóxico e no desenvolvimento da criança em longo alcance. Deste modo, os cuidadores parentais, educadores e profissionais da saúde devem reconhecer o impacto gerado pelo bullying na primeira infância e, assim, incrementar medidas de proteção e prevenção, que são essenciais tanto em contextos familiares quanto na área da saúde e da educação.

Palavras-chave: Criança; Bullying; Desenvolvimento infantil. 
http://dx.doi.org/10.5902/1984686X61983

\section{ABSTRACT}

Identify available scientific evidence about on the repercussions of bullying in early childhood. Integrative literature review that analyzed 13 articles from the PUBMED/MEDLINE, LILACS, CINAHL, Web of Science and PSYCOINFO databases based on the keywords child, bullying and child development, in the period 2009 to 2019, in Portuguese and English. The information was grouped into the thematic categories: Bullying in early childhood: relational and contextual aspects; Adverse parenting and aggressive interactions; Bullying in children in specific circumstances. Issues involving bullying in early childhood can have repercussions related to the process of toxic stress and the long-term development of the child. Thus, parental caregivers, educators and health professionals must recognize the impact generated by bullying in early childhood and, thus, increase protection and prevention measures, which are essential both in family contexts and in the area of health and education.

Keywords: Child; Bullying; Child development.

\section{RESUMEN}

Identificar evidencias científicas sobre como repercusiones del bullying en la primera infancia. Revisión de literatura integradora que analizó 13 artículos de las bases de datos PUBMED/MEDLINE, LILACS, CINAHL, Web of Science y PSYCOINFO en base a las palabras clave niño, bullying y desarrollo infantil, en el período 2009 a 2019, en portugués e inglés. La información se agrupa en las categorías temáticas: Bullying en la primera infancia: aspectos relacionados con el contexto; Crianza adversa e interacciones agresivas; Bullying en niños en circunstancias específicas. Los problemas relacionados con el acoso en la primera infancia pueden tener repercusiones relacionadas con el proceso de estrés tóxico y el desarrollo a largo plazo del niño. Así, los padres cuidadores, educadores y profesionales de la salud deben reconocer el impacto que genera el acoso en la primera infancia y, así, incrementar las medidas de protección y prevención, fundamentales tanto en los contextos familiares como en el ámbito de la salud y la educación.

Palabras clave: Niño; Bullying; Desarrollo infantil.

\section{Introdução}

A primeira infância, etapa do nascimento aos seis anos de idade, é um período de extrema relevância para o desenvolvimento humano, significativo para formar as estruturas e funções cerebrais que possibilitam o aperfeiçoamento futuro de habilidades complexas (SHONKOFF, 2011). Os primeiros anos de vida da criança são fundamentais para seu desenvolvimento físico, emocional, cognitivo e cultural (SHONKOFF, 2011).

O desenvolvimento infantil é um processo que envolve o crescimento físico, a maturação neurológica, comportamental, cognitiva, social e afetiva, e se ocorrer em circunstâncias adequadas gera competências às crianças para responder às necessidades 
http://dx.doi.org/10.5902/1984686X61983

individuais e sociais, considerando o seu contexto de vida (MOORE et al., 2017). Por sua vez, é construído a partir das interações com outras pessoas e é dependente de relações e ambientes sócio afetivos qualificados e estimulantes, o que contribui para 0 desenvolvimento da sua competência social, o seu bem-estar, o aprendizado e a apreensão de novas habilidades (SHONKOFF; BOYCE; MCEWEN, 2009; SHONKOFF, 2011).

Evidências científicas apontam que uma criança sem acesso a cuidadores sensíveis e atenciosos apresenta risco maior de ativação permanente das respostas do cérebro ao estresse (SHONKOFF; BOYCE; MCEWEN, 2009; SHONKOFF, 2011). A frequência de eventos como este pode prejudicar os circuitos neurais responsáveis pela aprendizagem e pela memória e levar a uma maior suscetibilidade a doenças relacionadas ao estresse, que afetam tanto a saúde física quanto mental (SHONKOFF; BOYCE; MCEWEN, 2009).

O aprofundamento da qualidade das relações e do desenvolvimento da vida social é de extrema importância para o ser humano. Nesse sentido, cabe apontar a necessidade de ampliar o entendimento do desenvolvimento social da criança quando outros fatores são agregados, como as situações que envolvem o bullying nos primeiros anos de vida.

O fenômeno do bullying é caracterizado como um processo de violência, multidimensional e relacional entre pares, que pode ocorrer de forma intencional, repetitiva e sem motivação aparente, demarcado pelo desequilíbrio e abuso sistemático de poder entre agressor e vítima, e amplamente prevalente no contexto escolar (OLWEUS, 2013; THOMAS et al., 2017). Pode ser tipificado em relação à ocorrência física (bater, chutar, beliscar), verbal (humilhar, apelidar, xingar, zoar, insultar), moral (difamar, discriminar, disseminar fofocas ou mentiras), psicológica (intimidar, ameaçar, perseguir, excluir), virtual ou cyberbullying (difamar, humilhar, zoar, insultar, ofender através da internet ou de outras tecnologias relacionadas ao mundo virtual). E os papeis desempenhados diante do bullying podem ser classificados em vítimas, agressores, observadores ou testemunhas e vítimasagressores (SCHULTZ et al., 2012; OLWEUS, 2013).

Alguns problemas psicossociais vinculados às situações do bullying já foram descritos em crianças, que ficam suscetíveis a desenvolver inquietação, ansiedade, depressão, pânico, insônia, estresse, ideação suicida, dor de cabeça, problemas gástricos, entre outros, trazendo danos à saúde física, mental e interferindo em seu pleno desenvolvimento (OLWEUS, 2013; IBEZIAKO et al., 2016). Estudos com adolescentes vítimas de bullying mostraram maiores taxas de tabagismo, etilismo, drogadição e comportamento suicida (FLEMING; JACOBSEN, 2010; BARSILAY et al., 2017). Essa sequência de distúrbios foi 
http://dx.doi.org/10.5902/1984686X61983

apontada como fator que interfere no processo ensino-aprendizado e diretamente na saúde desses indivíduos (MELLO et al., 2017).

Crianças e adolescentes vítimas de bullying podem apresentar mais sintomas emocionais, dificultando o desenvolvimento de suas habilidades sociais até a idade adulta (KONTAK et al., 2019). Outrossim, as habilidades sociais contribuem para que o indivíduo adquira competência para o desenvolvimento de tarefas, resolução de conflitos, controle emocional, e melhoria na aceitação pelos pares (SILVA et al., 2018). Além disso, pesquisadores indicam que a redução das ocorrências de bullying pode ocorrer a partir do trabalho conjunto entre família-escola, respeito aos direitos de todos, criação de diferentes espaços para crianças e adolescentes se expressarem, combate a qualquer tipo de discriminação, estímulo às relações seguras, estáveis e amparadoras entre crianças-paiscuidadores, e pelo desenvolvimento das habilidades de vida em crianças e adolescentes (OLWEUS, 1993; ZYCH et al., 2017).

No Brasil, a Pesquisa Nacional de Saúde do Escolar (PeNSE), realizada no ano de 2015, evidenciou que estudantes vítimas de bullying apresentavam maior prevalência de insônia, solidão, menos amigos e faltavam mais às aulas em comparação com os estudantes não vítimas (SILVA et al., 2017).

Conhecer e compreender as repercussões do bullying na primeira infância também contribui para a implementação de intervenções contextualizadas e mais eficazes. Estudos demostram que intervenções que englobam diferentes perspectivas sociais, educacionais, familiares e individuais, ou seja, que além da abordagem individual, incluem as famílias, as equipes interprofissionais e sua formação, e o suporte individual e coletivo para os envolvidos, têm maior efetividade (FREIRE; AIRES, 2012; SILVA et al., 2017). Assim, o bullying pode se constituir em um fator que afeta negativamente o desenvolvimento saudável de crianças e adolescentes, especialmente das vítimas, o que torna imprescindível a sua compreensão, especialmente na infância.

Dados do National Center for Education Statistics (2016) mostram que uma a cada cinco crianças ou adolescentes, entre 12 e 18 anos, relataram ter sido vítimas de bullying, todavia, para as crianças de menor idade, esses dados tornam-se mais desafiadores, considerando as dificuldades para sua identificação ainda na primeira infância. Frente à relevância que os primeiros anos de vida têm para o desenvolvimento de habilidades sociais, cabe identificar elementos apontados na literatura científica de situações adversas precoces que podem configurar bullying na primeira infância, já que o tema vem sendo mais 
http://dx.doi.org/10.5902/1984686X61983

investigado em crianças em idade escolar e adolescentes. Assim, é relevante o aprofundamento do tema, com o propósito de analisar lacunas dos conhecimentos científicos e apreender evidências relevantes para a promoção do desenvolvimento adequado na primeira infância.

O presente estudo objetivou identificar evidências científicas sobre as repercussões do bullying na primeira infância.

\section{Método}

Este estudo configura uma revisão integrativa, como um método de pesquisa constituído em análise da literatura científica sobre o bullying na primeira infância.

A revisão integrativa é um método que requer critérios na seleção da literatura a ser incluída na revisão, um processo estruturado com análise crítica dos resultados, visando ampliar a compreensão de um tema particular. Esse tipo de revisão inclui seis etapas: identificação do tema e seleção da questão de pesquisa, estabelecimento de critérios de inclusão e exclusão de estudos/busca na literatura, categorização dos dados, avaliação dos estudos incluídos na revisão, interpretação dos resultados e apresentação da revisão/síntese do conhecimento (MENDES; SILVEIRA; GALVÃO, 2008).

A primeira etapa da revisão abordou a definição do problema, com a identificação do tema e seleção da questão da pesquisa, utilizando-se a estratégia PICo - acrônimo que representa "Paciente", "Intervenção" e "Comparação/Desfecho". Dessa forma, para designar paciente ou problema (P): Criança; para intervenção (I): Desenvolvimento Infantil; e para desfecho (Co): Bullying. Assim, a questão norteadora adotada foi: "Quais são as evidências disponíveis na literatura científica sobre o bullying na primeira infância e suas repercussões no desenvolvimento de crianças menores de seis anos de idade?".

Para garantir a qualidade da pesquisa, foram selecionadas bases de dados com confiabilidade científica, elegendo-se: PubMed/MEDLINE, LILACS, CINAHL, Web of Science e PSYCOINFO. Para a busca foram utilizadas as palavras-chave: criança, desenvolvimento infantil, child, child development e bullying, combinadas de diferentes formas e realizadas em seis buscas diferentes.

$\mathrm{Na}$ definição da estratégia de busca, foi realizada a combinação dos operadores booleanos por meio dos conectores "AND" (combinação restritiva) com as palavras-chave relacionadas aos elementos da estratégia PICo, para ampliar o levantamento nas bases de dados e truncamentos para compor o cruzamento das buscas. Foram utilizadas as 
http://dx.doi.org/10.5902/1984686X61983

seguintes combinações: child AND bullying, criança AND bullying e child AND bullying AND child development. Os filtros utilizados para a busca em cada base de dados estão descritos no Quadro 1.

Quadro 1 - Distribuição dos filtros utilizados para a busca dos artigos em cada base de dados. Ribeirão Preto, SP, Brasil, 2020.

\begin{tabular}{|c|c|}
\hline Base de Dados & Filtros \\
\hline Pubmed & $\begin{array}{l}\text { - Texto completo; } \\
\text { - Período } 2009 \text { e } 2019 ; \\
\text { - Idioma inglês ou português; } \\
\text { - Denominações: recém-nascido ( } 28 \text { dias), bebê (1-23 meses), criança pré-escolar ( } 2 \\
\text { a } 5 \text { anos de idade) }\end{array}$ \\
\hline LILACS & - Sem opção de filtros \\
\hline CINAHL & $\begin{array}{l}\text { - Texto completo; } \\
\text { - Período } 2009 \text { e } 2019 ; \\
\text { - Revista acadêmica; } \\
\text { - Idade: child e preschool (2-5 years), infant (1-23 months), newborn (birth-1 month); } \\
\text { - Idioma inglês }\end{array}$ \\
\hline Web of Science & $\begin{array}{l}\text { - Período } 2009 \text { e 2019; } \\
\text { - Idioma inglês; } \\
\text { - Tipo de documento: artigo e acesso aberto }\end{array}$ \\
\hline PSYCOINFO & $\begin{array}{l}\text { - Idade neonatal (nascimento-1 mês), infância (2-23 meses), pré-escolar (2-5 anos); } \\
\text { - Tipo de documento: artigo de jornal/periódico/revista científica); } \\
\text { - Período } 2009 \text { e } 2019\end{array}$ \\
\hline
\end{tabular}

Fonte: dados da pesquisa (2020).

Para a seleção dos estudos, foram aplicados os critérios de inclusão: resumos e artigos disponíveis; idioma de publicação português e inglês, entre 2009 e 2019; e crianças menores de seis anos de idade. Excluíram-se os estudos que não apresentava resumo disponível e acesso online livre na íntegra; não ter em seus resultados contribuições para as perguntas estabelecidas na revisão; não possuir o caminho metodológico claramente descrito, revisões de literatura, dissertações, teses, livros e capítulos de livros.

A delimitação do período de busca justifica-se por seu objeto de investigação, que sofre transformações continuamente, e por considerar a relevância de incluir os estudos originais publicados mais recentemente e ainda pouco explorados. Assim, foram incluídos nesta revisão estudos originais, publicados na íntegra em periódicos científicos, revisados por pares, com crianças menores de seis anos de idade, abordagem sobre bullying na primeira infância e que apresentaram a repercussão do bullying ao desenvolvimento da criança. Abordagens quantitativas e qualitativas foram consideradas com o objetivo de abranger a diversidade da repercussão que o bullying traz nesta faixa etária.

A seleção dos estudos foi realizada no período de janeiro a agosto de 2020, por dois pesquisadores. Os estudos que atenderam os critérios de inclusão foram submetidos à 
leitura minuciosa destacando a temática abordada, ano de publicação, idioma, objetivos, metodologia, resultados e conclusões. A análise dos estudos originais foi fundamentada nos seguintes itens: conter questão da pesquisa, fundamentação teórico-metodológica, justificativa e relevância do estudo, aporte da literatura científica de outras pesquisas já realizadas, metodologia do estudo pertinente, seleção dos participantes, respostas à questão da pesquisa, e apontamentos de futuras pesquisas (BEYEA; NICOLL, 1998). Desse modo, os estudos selecionados responderam a estes itens e foram incluídos na presente revisão.

Em situação de desacordos entre os revisores em relação aos dados extraídos, foi acionado um terceiro pesquisador para avaliar a inclusão ou não do estudo, tendo como referência a publicação original. Em seguida, foram realizadas as etapas de categorização e identificação, e foi elaborada a discussão crítica dos resultados, para compor as conclusões quanto ao objeto de estudo e limitações.

\section{Resultados}

Nas buscas em todas as bases de dados, foram obtidos 1.586 artigos para a pesquisa. Foram excluídas 482 por duplicações, ficando então 1.104 estudos para as etapas de seleção. Pela triagem do título e análise dos resumos, foram excluídos 1.061 estudos, sendo 107 por não estarem disponíveis nos idiomas inglês e/ou português, 75 por apresentarem no título o método de revisão da literatura, 458 estudos pela faixa etária além da primeira infância, 412 artigos por apresentarem assuntos distintos ao bullying na primeira infância, seis eram livros e/ou capítulos de livros e três estudos por apresentaram no resumo os anos fora do período entre 2009 e 2019.

A partir dessa etapa, foram selecionados 43 estudos para a leitura na íntegra. A Figura 1 apresenta as informações referentes a cada etapa de obtenção de dados, de acordo com as recomendações da ferramenta Preferred Reporting Items for Systematic Reviews and Meta-Analyses (PRISMA) (MOHER et al., 2009). 
http://dx.doi.org/10.5902/1984686X61983

Figura 1 - Fluxograma PRISMA dos artigos encontrados nas bases de dados PUBMED/MEDLINE, LILACS, CINAHL, Web of Science E PSYCOINFO. Ribeirão Preto, SP, Brasil, 2020.
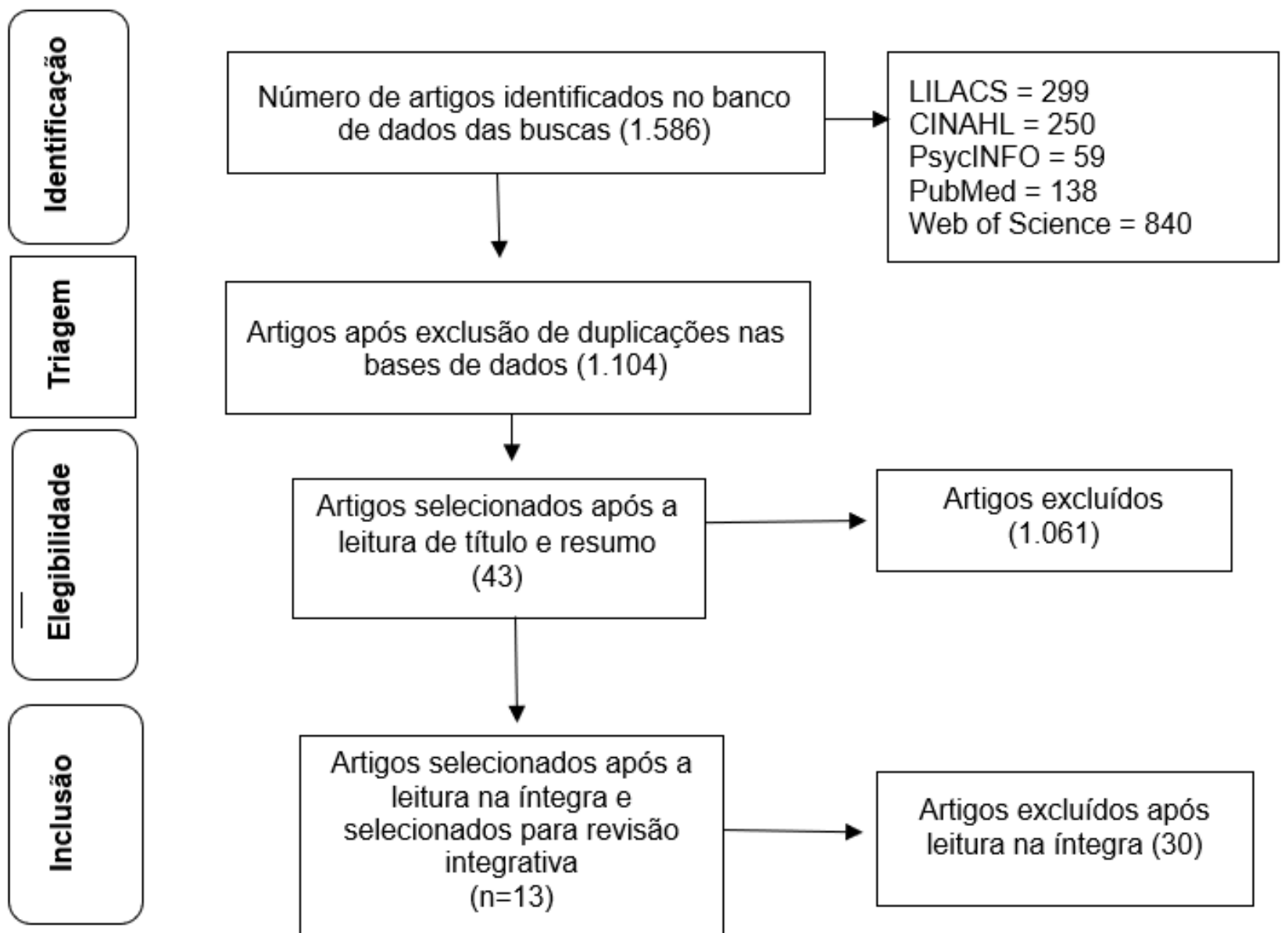

Artigos após exclusão de duplicações nas bases de dados (1.104)

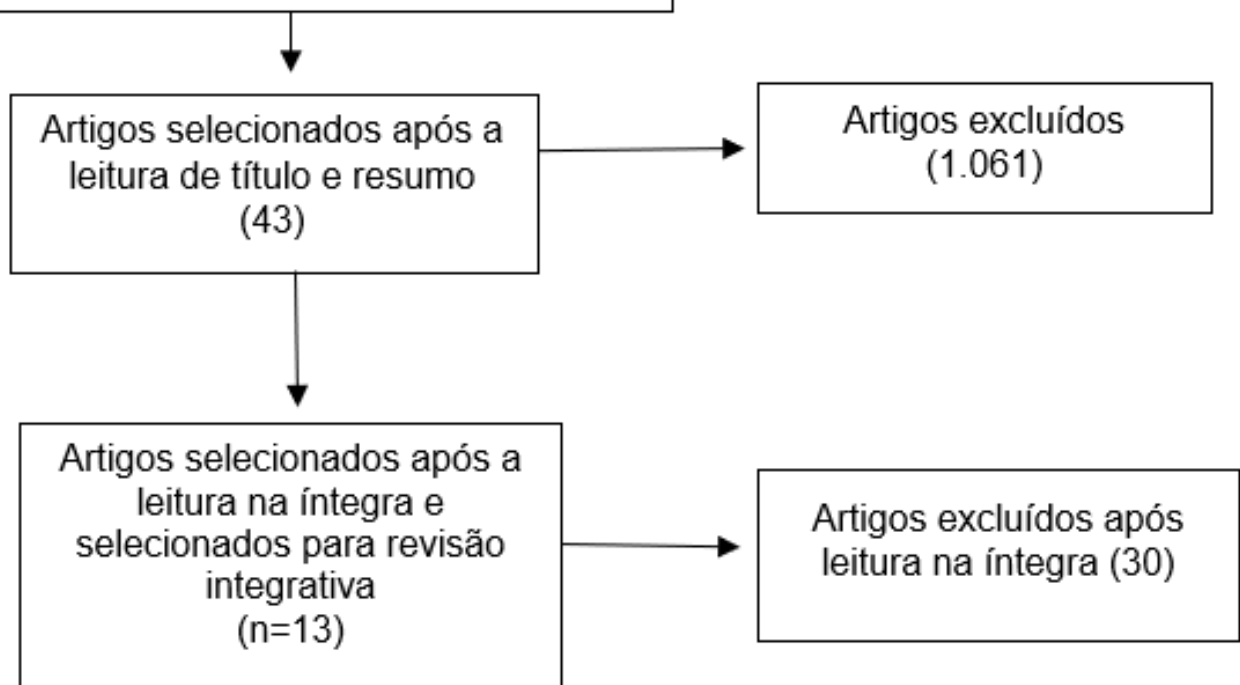

Fonte: dados da pesquisa (2020).

Dos 43 estudos, 30 foram excluídos, sendo 15 por serem com crianças acima de seis anos de idade, quatro que retratam o bullying na perspectiva dos professores, um estudo por analisar a lógica penal da vitimização do bullying, nove estudos por não estarem voltados para a questão norteadora da pesquisa e uma investigação por se tratar de estudo de reflexão, não identificado no resumo. Assim, 13 estudos foram selecionados para análise. O Quadro 2 apresenta uma síntese dos estudos selecionados para esta revisão integrativa. 
http://dx.doi.org/10.5902/1984686X61983

Quadro 2 - Distribuição dos estudos selecionados de acordo com título, autores, país, objetivos e método. Ribeirão Preto, SP, Brasil, 2020.

(continua)

\begin{tabular}{|c|c|c|c|c|}
\hline Título & Autores & País & Objetivo & Método \\
\hline $\begin{array}{l}\text { 1.Warning signs of preschool } \\
\text { victimization using the } \\
\text { strengths and difficulties } \\
\text { questionnaire: prevalence } \\
\text { and individual and family risk } \\
\text { factors }\end{array}$ & $\begin{array}{l}\text { NAVARRO et al., } \\
2019\end{array}$ & Espanha & $\begin{array}{l}\text { Relatar a prevalência e os fatores de } \\
\text { risco de sinais de alerta de } \\
\text { vitimização na pré-escola, conforme } \\
\text { avaliado pelo SDQ, um questionário } \\
\text { de triagem amplamente utilizado } \\
\text { relatado por pais e professores. }\end{array}$ & $\begin{array}{l}\text { Estudo } \\
\text { Longitudinal }\end{array}$ \\
\hline $\begin{array}{l}\text { 2.Mothers' difficulties and } \\
\text { expectations for intervention } \\
\text { of bullying among young } \\
\text { children in South Korea }\end{array}$ & LEE; JU, 2019 & $\begin{array}{l}\text { Coreia do } \\
\text { Sul }\end{array}$ & $\begin{array}{l}\text { Investigar as dificuldades das mães } \\
\text { em lidar com o bullying de seus filhos } \\
\text { e suas expectativas em relação à } \\
\text { intervenção de bullying para crianças } \\
\text { pequenas na Coreia do Sul. }\end{array}$ & $\begin{array}{l}\text { Abordagem } \\
\text { Qualitativa }\end{array}$ \\
\hline $\begin{array}{l}\text { 3.Peer-victimization of young } \\
\text { children with developmental } \\
\text { and behavioral difficulties-a } \\
\text { population-based study }\end{array}$ & $\begin{array}{l}\text { OKSENDAL et al., } \\
2019\end{array}$ & Noruega & $\begin{array}{l}\text { Investigar se crianças pequenas } \\
\text { com dificuldades de } \\
\text { desenvolvimento e comportamento } \\
\text { têm maior risco de vitimização por } \\
\text { pares em comparação com crianças } \\
\text { com desenvolvimento típico. }\end{array}$ & $\begin{array}{l}\text { Estudo } \\
\text { coorte }\end{array}$ \\
\hline $\begin{array}{l}\text { 4.Children's voices on } \\
\text { bullying in Kindergarten }\end{array}$ & $\begin{array}{l}\text { HELGELAND; } \\
\text { LUND, } 2017\end{array}$ & Noruega & $\begin{array}{l}\text { Examinar e discutir o bullying no } \\
\text { jardim de infância. }\end{array}$ & $\begin{array}{l}\text { Abordagem } \\
\text { Qualitativa }\end{array}$ \\
\hline $\begin{array}{l}\text { 5.Peer victimization and peer } \\
\text { rejection during early } \\
\text { childhood }\end{array}$ & $\begin{array}{l}\text { GOLDLESKI et al., } \\
2015\end{array}$ & $\begin{array}{l}\text { Estados } \\
\text { Unidos da } \\
\text { América }\end{array}$ & $\begin{array}{l}\text { Explorar lacunas e limitações sobre } \\
\text { vitimização e rejeição por pares e, } \\
\text { em particular, as associações } \\
\text { prospectivas entre vitimização } \\
\text { relacional e física e rejeição de pares } \\
\text { de } 3 \text { a } 5 \text { anos de idade. }\end{array}$ & $\begin{array}{l}\text { Estudo } \\
\text { Longitudinal } \\
\text { curto prazo }\end{array}$ \\
\hline $\begin{array}{l}\text { 6.Parenting style influences } \\
\text { bullying: a longitudinal study } \\
\text { comparing children with and } \\
\text { without behavioural problems }\end{array}$ & $\begin{array}{l}\text { RAJENDRAN; } \\
\text { KRUSZEWSKI; } \\
\text { HALPERIN, } 2015\end{array}$ & $\begin{array}{l}\text { Estados } \\
\text { Unidos da } \\
\text { América }\end{array}$ & $\begin{array}{l}\text { Examinar diferenças no bullying em } \\
\text { crianças com Transtorno de Déficit } \\
\text { de Atenção e Hiperatividade e } \\
\text { Transtorno Desafiador de Oposição } \\
\text { e crianças sem esses transtornos. }\end{array}$ & $\begin{array}{l}\text { Estudo } \\
\text { Longitudinal } \\
\text { prospectivo }\end{array}$ \\
\hline $\begin{array}{l}\text { 7.With whom and where you } \\
\text { play: preschoolers' social } \\
\text { context predicts peer } \\
\text { victimization }\end{array}$ & $\begin{array}{l}\text { ANDREWS et al., } \\
2014\end{array}$ & $\begin{array}{l}\text { Estados } \\
\text { Unidos da } \\
\text { América }\end{array}$ & $\begin{array}{l}\text { Examinar características do contexto } \\
\text { social da criança em idade pré- } \\
\text { escolar (tamanho do grupo de } \\
\text { brincadeiras, composição de gênero } \\
\text { dos grupos de brincadeiras e } \\
\text { ambiente de brincadeiras) que } \\
\text { influenciam mudanças na } \\
\text { vitimização de crianças em idade } \\
\text { pré-escolar. }\end{array}$ & $\begin{array}{l}\text { Estudo } \\
\text { Longitudinal } \\
\text { curto prazo }\end{array}$ \\
\hline
\end{tabular}


Quadro 2 - Distribuição dos estudos selecionados de acordo com título, autores, país, objetivos e método. Ribeirão Preto, SP, Brasil, 2020.

(conclusão)

\begin{tabular}{|c|c|c|c|c|}
\hline $\begin{array}{l}\text { 8. Teacher and peer reports of } \\
\text { Overweight and bullying } \\
\text { among young primary school } \\
\text { children }\end{array}$ & $\begin{array}{l}\text { JANSENS et al., } \\
2014\end{array}$ & Holanda & $\begin{array}{l}\text { Examinar se o sobrepeso ou a } \\
\text { obesidade estão associados à } \\
\text { vitimização e à prática de bullying em } \\
\text { crianças de } 5 \text { a } 6 \text { anos de idade nas } \\
\text { primeiras séries do ensino } \\
\text { fundamental. }\end{array}$ & $\begin{array}{l}\text { Estudo } \\
\text { Transversal }\end{array}$ \\
\hline $\begin{array}{l}\text { 9.The relationship between } \\
\text { emotional competence and } \\
\text { hostile/prosocial behavior in } \\
\text { Albanian preschoolers: An } \\
\text { exploratory study }\end{array}$ & $\begin{array}{l}\text { FARINA; } \\
\text { BELACCHI, } 2014\end{array}$ & Albânia & $\begin{array}{l}\text { Explorar as relações entre a } \\
\text { competência socioemocional dos } \\
\text { pré-escolares albaneses e sua } \\
\text { tendência atribuída a assumir papéis } \\
\text { pró-sociais e/ou hostis. }\end{array}$ & $\begin{array}{l}\text { Estudo } \\
\text { controlado } \\
\text { randomizado }\end{array}$ \\
\hline $\begin{array}{l}\text { 10.Aggressive interactions } \\
\text { during free-play at preschool } \\
\text { of children with and without } \\
\text { developmental coordination } \\
\text { disorder }\end{array}$ & $\begin{array}{l}\text { KENNEDY- } \\
\text { BEHR; RODGER; } \\
\text { MICKAN, } 2013\end{array}$ & Alemanha & $\begin{array}{l}\text { Explorar e comparar os incidentes de } \\
\text { agressão e vitimização durante o } \\
\text { brincar livre na pré-escola de } \\
\text { crianças com e sem transtorno de } \\
\text { coordenação no desenvolvimento. }\end{array}$ & $\begin{array}{l}\text { Estudo quase- } \\
\text { experimental }\end{array}$ \\
\hline 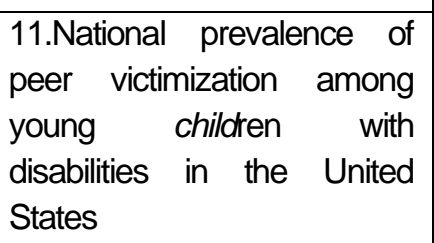 & $\begin{array}{l}\text { SON; PARISH; } \\
\text { PETERSON, } \\
2012\end{array}$ & $\begin{array}{l}\text { Estados } \\
\text { Unidos da } \\
\text { América }\end{array}$ & $\begin{array}{l}\text { Analisar uma amostra nacional e } \\
\text { representativa para examinar a } \\
\text { vitimização por pares de crianças } \\
\text { pequenas com deficiência }\end{array}$ & $\begin{array}{l}\text { Estudo de } \\
\text { prevalência }\end{array}$ \\
\hline $\begin{array}{l}\text { 12.Prevenção da violência } \\
\text { escolar: avaliação de um } \\
\text { programa de intervenção }\end{array}$ & MENDES, 2011 & Portugal & $\begin{array}{l}\text { Avaliar os resultados de um } \\
\text { programa anti-violência escolar, } \\
\text { implementado em } 307 \text { crianças do } \\
\text { segundo ciclo de uma escola de } \\
\text { Lisboa. }\end{array}$ & $\begin{array}{l}\text { Estudo de } \\
\text { pesquisa-ação }\end{array}$ \\
\hline $\begin{array}{l}\text { 13.Individual differences in } \\
\text { the development of early peer } \\
\text { aggression: Integrating } \\
\text { contributions of self- } \\
\text { regulation, theory of mind, } \\
\text { and parenting }\end{array}$ & $\begin{array}{l}\text { OLSON et al., } \\
2011\end{array}$ & $\begin{array}{l}\text { Estados } \\
\text { Unidos da } \\
\text { América }\end{array}$ & $\begin{array}{l}\text { Identificar preditores em idade pré- } \\
\text { escolar da agressão de crianças } \\
\text { após a transição para a escola. }\end{array}$ & $\begin{array}{l}\text { Estudo } \\
\text { Longitudinal } \\
\text { prospectivo }\end{array}$ \\
\hline
\end{tabular}

Fonte: dados da pesquisa (2020).

Após a extração dos dados, os resultados foram organizados, codificados, por meio da interpretação dos resultados dos estudos selecionados, originando as categorias temáticas: Bullying na primeira infância: aspectos relacionais e contextuais; Cuidados parentais adversos e interações agressivas; Bullying em crianças em circunstâncias específicas. 
http://dx.doi.org/10.5902/1984686X61983

\section{Bullying na primeira infância: aspectos relacionais e contextuais}

Os estudos selecionados apontaram divergências entre o sexo masculino/feminino e os níveis de agressividade, de comportamentos problemáticos e da frequência do bullying.

Crianças entre três e seis anos de idade apresentaram níveis mais altos de agressão, entre meninos quando comparados a meninas da mesma idade (OLSON et al., 2011). O referido estudo identificou, ainda que os meninos receberam mais punição corporal dos pais e possuem níveis mais baixos de compreensão do que meninas, tendo por base a teoria da mente. A teoria da mente está relacionada à habilidade das crianças de compreenderem seus próprios estados mentais e dos outros, e dessa maneira, predizem suas ações e comportamentos (OLSON et al., 2011).

Outro estudo também indicou que os meninos apresentam níveis mais altos em relação à vitimização e ao comportamento problemático em relação às meninas (ANDREWS et al., 2014). Todavia, os meninos apresentaram maior proporção de interações sociais entre pares, com uma comunicação mais efetiva e cooperação em atividades, para entender semelhanças e negociar diferenças (ANDREWS et al., 2014). Nesta investigação, a interação social foi verificada a partir da proposta de jogos em grupos, e para os meninos, esta estratégia revelou um efeito favorável, visto que passaram mais tempo jogando e interagindo entre pares, e consequentemente, houve redução da vitimização.

Embora neste estudo o sexo tenha sido explorado como um fator que pudesse potencializar o bullying, suas análises preliminares sugeriram que este aspecto não foi significante, e o que foi destacado referiu-se a importância de controlar os subtipos de vitimização física e de agressão, ao examinar as associações prospectivas únicas entre rejeição de pares e vitimização relacional (ANDREWS et al., 2014).

A associação entre vitimização relacional, definida por atos que prejudicam 0 relacionamento entre pares, como excluir de atividades, disseminar fofocas ou mentiras, difamação, comentários maldosos e críticos, e a rejeição por pares é preocupante por contribuir para o aumento da vulnerabilidade das crianças e para as experiências negativas, mesmo entre crianças muito pequenas (GODLESKI et al., 2015; NAVARRO et al., 2019). A rejeição por pares desempenha um papel importante na previsão de aumento na vitimização relacional, mesmo quando há controle da vitimização física (GODLESKI et al., 2015). 
http://dx.doi.org/10.5902/1984686X61983

Um estudo espanhol acrescentou a estes aspectos, a relação de fatores contextuais com o aumento da vitimização por pares, como pertencer a uma família de baixo status socioeconômico e a dificuldade inicial em fazer amigos (NAVARRO et al., 2019).

Outro estudo mostrou os aspectos referentes aos sentimentos que envolviam as crianças vítimas do bullying. Para elas, a exclusão das brincadeiras, principalmente por seus colegas ou melhores amigos, é o que Ihes causa mais temor, com sentimento de tristeza e frustração, mostrando o quanto essas crianças podem tornar-se vulneráveis no convívio entre os pares, com consequências futuras para o seu desenvolvimento (HELGELAND; LUND, 2017).

O detalhamento dos tipos de vitimização física e as características da agressão têm relevância para um exame mais minucioso da relação entre rejeição de pares e vitimização relacional (GODLESKI et al., 2015). Coadunando com estes aspectos, estudos ressaltam que os processos de inclusão e exclusão no jardim de infância são muito importantes (FARINA; BELACCHI, 2014; LEE; JU, 2019), o que sugere que devem ser amplamente pesquisados por meio de abordagens qualitativas e quantitativas, com informações dos funcionários, educadores, cuidadores parentais e crianças.

\section{Cuidados parentais adversos e interações agressivas}

Em relação à parentalidade, os estudos selecionados enfatizaram que a relação entre cuidadores parentais e filhos em situações de bullying pode impactar a agressividade na primeira infância e em outras etapas da vida.

Crianças pequenas que sofreram punição corporal pelos cuidadores parentais apresentaram repercussões na pré-escola, com interações agressivas entre pares (OLSON et al., 2011). Além disso, o sistema patriarcal pode promover uma atitude submissa nas meninas que limita o comportamento proativo manifesto, hostil ou pró-social (FARINA; BELACCHI, 2014).

Os comportamentos dos pais e mães jovens foram analisados a partir da responsividade materna agressiva e da punição corporal parental, por exemplo, palmadas, agarrões e agitações (OLSON et al., 2011). Quando os dois aspectos citados foram introduzidos simultaneamente, apenas o castigo corporal contribuiu para maiores taxas de agressão. Quando os níveis de agressão entre colegas na pré-escola foram controlados, foi observado que a receptividade agressiva da mãe contribuiu para agressão em idade 
escolar. A investigação mostrou que o castigo corporal foi estatisticamente significante para a agressividade das crianças (OLSON et al., 2011).

Outra questão a ser destacada é o contexto familiar, e particularmente, a falta de um dos cônjuges, que podem potencializar o ser agressor, sobretudo, de famílias monoparentais (MENDES, 2011; FARINA; BELACCHU, 2014). A monoparentalidade pode se constituir em fator de vulnerabilidade ao bullying na infância e adolescência, associada ao aumento do estresse familiar e situações adversas na família, influenciando no comportamento e desenvolvimento (MENDES, 2011; FARINA; BELACCHI, 2014).

Outro elemento investigado foi o apoio dos cuidadores parentais à autonomia da criança entre quatro e cinco anos de idade, que apresentou redução no bullying em comparação aos que não receberam ou receberam pouco apoio (RAJENDRAN; KRUSZEWSKI; HALPERIN, 2016). Tais resultados sugerem a relevância da construção da autonomia na infância no contexto familiar, para gerar contribuições de longo alcance.

\section{Bullying em crianças em circunstâncias específicas}

Situações relacionadas à imagem corporal, mental e comportamental podem influenciar e aumentar a frequência do bullying na primeira infância.

Em crianças com alto Índice de Massa Corporal (IMC) foi encontrado um aumento bullying físico, verbal e desprezo social, mais especificamente entre meninos (JANSEN et al., 2014). Os resultados do referido estudo sugerem que crianças obesas apresentam maior risco de serem vítimas do bullying do que as com peso adequado e, na questão de gênero, os meninos apresentaram mais risco de serem agredidos do que as meninas, de acordo com os relatos dos professores. Ainda, o estudo aponta que a perpetração do bullying foi analisada por uma interação entre IMC e sexo, em que um IMC mais alto previa níveis mais altos de bullying físico, verbal e relacional, mas não de bullying material e de vitimização.

Outras situações como os transtornos psiquiátricos e deficiências também foram investigadas. Em pré-escolares de três a cinco anos foram encontrados altos níveis de bullying em crianças diagnosticadas com transtornos psiquiátricos, com três vezes mais chances de serem classificadas como vítimas ou vítimas agressivas em relação a outras crianças (BELDEN; GAFFREY; LUBY, 2012). A referida investigação identificou que crianças diagnosticadas com distúrbios disruptivos, depressivos e em uso de ansiolíticos apresentaram seis vezes mais chances de se tornarem vítimas de agressão. 
http://dx.doi.org/10.5902/1984686X61983

Em relação às deficiências (visual, auditiva, emocional, mental, comportamental, dificuldades de aprendizagem, comprometimento ortopédico e de linguagem), um estudo demonstrou alta incidência de bullying, sendo que quase um terço das crianças participantes foram vítimas por pares e a prevalência de vitimização por pares aumentou conforme o passar dos três anos do estudo longitudinal (SON; PARISH; PETERSON, 2012). Neste estudo, nas crianças com três anos de idade, foi identificada a vitimização relacional como a mais comum e, quando completaram quatro e cinco anos a vitimização verbal foi a mais frequente. Os autores enfatizam que a vitimização de pares é um problema social sério, com implicações negativas ao desenvolvimento psicossocial das crianças.

Crianças com diagnóstico de Transtorno de Déficit de Atenção e Hiperatividade (TDAH) e Transtorno Desafiador de Oposição (TDO) também foram investigadas em outro estudo, e foram associadas com mais frequência a sofrerem os primeiros atos do bullying (ofensas e rejeição), além de possuir maior risco de assédio moral (RAJENDRAN; KRUSZEWSKI; HALPERIN, 2016). As mais propensas à intimidação foram as com TDAH e sem TDO, diferente das que possuem os dois diagnósticos, com mais propensão a sofrer assédio moral em relação a crianças sem esses diagnósticos.

Crianças com transtornos de coordenação do desenvolvimento e de comportamento também foram analisadas e foi encontrado que são mais expostas às agressões, inclusive as físicas proativas, como golpes, agarrões e chutes, do que as crianças sem estes diagnósticos (KENNEDY-BEHR; RODGER; MICKAN, 2013; OKSENDAL et al., 2019). Uma parcela das crianças entre quatro e seis anos de idade estava envolvida em uma interação agressiva, seja como agressor ou vítima (KENNEDY-BEHR; RODGER; MICKAN, 2013). Esses resultados indicam que as crianças com transtornos de coordenação do desenvolvimento e de comportamento foram envolvidas com mais frequência no bullying e estavam mais sujeitas a sofrerem ataque agressivo do que as crianças sem estes transtornos.

\section{Discussão}

Os resultados dos artigos científicos analisados nesta revisão evidenciam a relação do bullying na primeira infância com os aspectos emocionais e contextuais, os cuidados parentais e circunstâncias ligadas a interações agressivas, e situações do bullying em crianças obesas, com transtornos psiquiátricos, desenvolvimento e deficiências. 
http://dx.doi.org/10.5902/1984686X61983

Entre os aspectos contextuais, os estudos demonstraram que meninos na faixa etária entre três e seis anos de idade sofrem mais agressão, vitimização, proporção de comportamento problemático, quando comparados com meninas da mesma idade (OLSON et al., 2011; ANDREWS et al., 2014). Outro estudo, que incluiu crianças maiores, com idade média de 12 anos, também verificou que há maior vitimização pelos meninos em todos os tipos de bullying, sendo o psicológico o mais comum, em seguida o bullying físico e por último o bullying virtual (MARCOLINO et al., 2018). Todavia, há estudo (SILVA; DASCANIO; VALLE, 2016) que contradiz estes desfechos, visto que encontrou a situação de ser vítima de bullying mais em meninas.

A respeito de cometer alguma agressão física contra os colegas, pelo menos uma vez, estudo realizado com crianças em outra faixa etária, entre oito e 16 anos, constatou que os meninos tiveram maior participação, já o bullying verbal é o mais frequente entre meninas (ZEQUINÃO et al., 2016). Outro estudo identificou que o bullying sofrido pelos meninos e meninas entre 12 e 15 anos foi diferente, as meninas apresentaram formas mais veladas de agressividade, como a fofoca, e os meninos foram mais agressivos fisicamente (SILVA; DASCANEO; VALLE, 2016).

Em relação aos cuidados parentais, a literatura científica analisada indica que o uso de castigos físicos ou medidas disciplinares severas são ligadas às situações de bullying na infância. Crianças que sofrem punição corporal dos pais tendem a manifestar interações agressivas com seus colegas na pré-escola (KNOUS-WESFALL et al., 2012). Cuidadores parentais que dão suporte aos filhos vítimas de bullying, compartilham ideias, apoiam na tarefa escolar e conversam com os filhos, podem contribuir para romper o ciclo de violência e abusos, e a desenvolver mecanismos de enfrentamento (LEE; JU, 2019).

Interações familiares positivas podem se constituir em fator de proteção em situação de vitimização ao bullying. Por outro lado, interações negativas podem potencializar a vulnerabilidade de envolvimento seja como agressores, vítimas e vítimas-agressores, uma vez que suas experiências no contexto e interação familiar irão influenciar no seu desenvolvimento social (OLIVEIRA et al., 2020). A organização e a relação familiar podem influenciar a construção de habilidades e respostas sociais, que podem potencializar ou reduzir a vulnerabilidade em relação ao bullying, seja enquanto vítima ou agressor (OLIVEIRA et al., 2018).

Um estudo com crianças e adolescentes na idade entre 10 e 17 anos mostrou que pais que possuem vínculos com os amigos dos filhos têm menos chances de se envolverem 
http://dx.doi.org/10.5902/1984686X61983

em situações de bullying (SHETGIRI; LIN; FLORES, 2013). Ademais, outra investigação verificou que a supervisão de adultos pode contribuir para a introspecção sobre a dificuldade de lidar e construir relacionamentos sociais e grupais (CUERVO; MARTINEZ; ACUNÃ, 2012). Portanto, é muito importante a mediação na participação dos cuidadores parentais na vida dos filhos, sem prejuízos ao desenvolvimento e às interações sociais da criança.

A família desenvolve um papel fundamental na construção da personalidade e comportamento do indivíduo, pois sua relação intrafamiliar influencia sobremaneira nas ações tomadas pelas crianças e adolescentes no meio externo, e o ambiente familiar contribui muito na sua conduta frente ao bullying (SILVA; ARAÚJO, 2015). Em geral, os agressores e/ou vítimas tendem a ter relações parentais endurecidas, pais autoritários, com menos sentimentos de envolvimento, empatia familiar e maiores níveis de castigos punitivos, considerados pouco assertivos, podendo ter maior vulnerabilidade de se envolverem em situações de bullying (FARIA, 2020).

Investigações ressaltam que contextos familiares desfavoráveis e/ou com um estilo parental negligente e com poucas regras, deixando a criança de lado ou se for autoritário com recurso a punições físicas, as probabilidades de potencializar uma reação/ação desajustada da criança são altas, sendo mais provável a criança envolver-se em situações de bullying (ALMEIDA; FOFONKA; WEISS, 2017; OLIVEIRA et al., 2020).

Deste modo, é possível observar que relacionamentos e dinâmicas familiares protetivos, proativos e positivos podem contribuir para o não envolvimento em situações de bullying, em contraposição aos relacionamentos fragilizados e conflituosos que podem gerar comportamentos de violência, a exemplo do bullying, que sugerem ter a finalidade de superar e ou manifestar suas frustrações e conflitos relacionados à vida familiar e social.

No contexto do bullying por circunstâncias específicas, um estudo retrata, por meio de uma revisão da literatura, que o bullying principalmente contra crianças com necessidades especiais está se tornando cada vez mais frequente nas escolas, expondo mais as vulnerabilidades de seu estado de saúde como alvos fáceis (SANTOS; LOPES, 2017). Esses aspectos são semelhantes aos relatados nos estudos analisados na presente revisão (SON; PARISH; PETERSON, 2012; KENNEDY-BEHR; RODGER; MICKAN, 2013; RAJENDRAN; KRUSZEWSKI; HALPERIN, 2016; OKSENDAL et al., 2019), o que sugere a necessidade de atenção nas escolas para evitar situações constrangedoras e contribuir para que as crianças não se afastem da escola. 
http://dx.doi.org/10.5902/1984686X61983

$\mathrm{Na}$ escola, as crianças desenvolvem os primeiros relacionamentos além da sua família. Para tanto, é necessário que a instituição escolar saiba estabelecer comparativos para distinção do bullying e de outras violências escolares, valorizando a solidariedade e a tolerância, para que tais valores sejam trabalhados com os alunos, mesmo quando pequenos (SILVA; ARAÚJO, 2015; NAVARRO et al., 2019).

Neste sentido, é imprescindível a reflexão sobre o papel e a interferência dos diferentes contextos de socialização, a exemplo da família, da escola e da sociedade, na emergência de comportamentos agressivos. Na realidade brasileira, as relações estabelecidas na comunidade escolar no desenvolvimento das atividades pedagógicas estão permeadas, de modo contundente, por variadas formas de violência, reproduzindo a vida social extramuros escolar. A violência escolar tem suscitado investigações, não só em função da contradição que ela representa em relação à missão educativa da escola, mas pelas consequências a longo prazo que dela podem decorrer.

Estudo sobre o estresse na infância (SHONKOFF; GARNER, 2012) aponta que existem três tipos de estresse: estresse positivo: caracterizado como um estresse normal, fundamental para o desenvolvimento da criança, com algumas elevações da frequência cardíaca; estresse tolerável: algumas dificuldades que são suportáveis, limitada no tempo e necessitam do auxílio de um adulto para que a criança consiga se adaptar, havendo a recuperação do cérebro e outros órgãos nas quais poderiam ser prejudiciais; estresse tóxico: quando a criança vivencia situações adversas constantes e prolongadas, tais como abuso físico, negligência e violência. Essas situações podem afetar o desenvolvimento da arquitetura do cérebro e outros órgãos do corpo, aumentando o risco de doenças no decorrer dos anos (SHONKOFF; GARNER, 2012). Nesse sentido, as questões que envolvem o bullying na primeira infância podem estar muito atreladas ao processo de estresse tóxico e suas repercussões no desenvolvimento da criança em longo alcance.

Para além deste aspecto, há que se problematizar o fato das crianças estarem presenciando, ainda na infância, enquanto observadoras, as ocorrências de bullying e a consequente aprendizagem por observação, uma vez que expostas a este modelo de violência entre pares, de forma repetitiva, seu comportamento pode ser afetado. Esse caminho pode facilitar a reprodução de um comportamento aprendido e, ao longo do seu desenvolvimento, internalizar o comportamento agressivo como forma de resolver conflitos e de se empoderar. 
http://dx.doi.org/10.5902/1984686X61983

Assim, as medidas de proteção e prevenção do bullying são essenciais, tanto em contextos familiares quanto da área da saúde e da educação (LEE; JU, 2019). Promover as habilidades sociais na primeira infância é de extrema importância para fortalecer as práticas antibullying assim como o incremento das ações intersetoriais - escola e saúde para potencializar a qualidade de vida, da saúde e do aprendizado, contribuindo para a construção da autonomia e das interações sociais.

\section{Conclusão}

A presente revisão analisou 13 artigos científicos distribuídos em três continentes, e trouxe evidências importantes sobre as repercussões do bullying, particularmente para a faixa etária entre os três e seis anos de idade.

O bullying foi mais identificado em meninos do que meninas da mesma idade e a rejeição por pares desempenhou papel importante no aumento da vitimização das crianças. Outro aspecto elencado foi o contexto da parentalidade, em que crianças que sofrem castigo corporal por parte dos cuidadores parentais apresentaram maiores índices de interações agressivas entre pares.

Em contextos de apoio parental e estímulo à autonomia das crianças há indicativos de redução do bullying na primeira infância, sugerindo que o ambiente que a criança está inserida e as interações estabelecidas repercutem no seu desenvolvimento. O bullying foi também caracterizado em circunstâncias específicas, em crianças com IMC elevado e com transtornos psiquiátricos, psicológicos ou deficiências, que apresentaram mais chances de serem vítimas de agressão em relação às outras crianças.

Ademais, o estudo trouxe a importância de investigar o bullying na primeira infância para apontar as repercussões para a vida das crianças e em longo alcance, visto que este período é de extrema relevância para o desenvolvimento humano. Neste contexto, há premência na obtenção de subsídios que permitam promover mais o desenvolvimento adequado na primeira infância, considerando que o bullying é um problema atual, desafiador e quando identificado oportunamente, desde os primeiros anos, pode ter grande impacto no desenvolvimento socioemocional.

Os cuidadores parentais, educadores e profissionais da saúde devem reconhecer as repercussões geradas pelo bullying enquanto estresse tóxico, na primeira infância e para a vida adolescente e adulta e, assim, incrementar as medidas de proteção e prevenção, que são essenciais tanto em contextos familiares quanto na área da saúde e da educação. 
http://dx.doi.org/10.5902/1984686X61983

As limitações deste estudo estão relacionadas à produção do conhecimento em sua maioria por países desenvolvidos e em nível internacional, reduzindo a análise de elementos para a realidade brasileira. Ao mesmo tempo, o delineamento de poucos estudos e de estudos qualitativos pode conter apreensão insuficiente do tema, pela falta de análises sobre percepções, significados e sentidos sobre o bullying na primeira infância. Cabe apontar que experiências relacionadas ao desenvolvimento na primeira infância têm implicações singulares e contextuais, considerando especificidades sociais, emocionais e culturais e, desse modo, novas pesquisas poderão trazer outros elementos que abordem o tema em diferentes conjunturas.

\section{Referências}

ALMEIDA, Loreni Dutra; FOFONKA, Luciana; WEISS, Claudia Suéli. Violência, ética, moral e bullying no espaço escolar. Revista Maiêutica - Estudos Linguísticos, Literários e Formação Docente. Indaial, v. 5, n. 1, p. 53-62, 2017. Disponível em: https://publicacao.uniasselvi.com.br/index.php/LED_EaD/article/view/1751/859. Acesso em: 09 jul. 2020.

ANDREWS, Naomi C. Z. et al. With whom and where you play: preschoolers' social context predicts peer victimization. Social Development. Oxford, v. 23, n. 2, p. 357-75, 2014. Disponível em: https://www.ncbi.nlm.nih.gov/pmc/articles/PMC4052953. Acesso em: 09 jan. 2020.

BARZILAY, Shira. et al. (2017). Bullying victimization and suicide ideation and behavior among adolescents in Europe: a 10-country study. Journal of Adolescent Health. New York, v. 61, n. 2, p. 179-86, 2017. Disponível em: https://doi.org/10.1016/j.jadohealth.2017.02.002. Acesso em: 23 dez. 2020.

BELDEN, Andy C.; GAFFREY, Michael S.; LUBY, Joan L. Relational aggression in children with preschool-onset psychiatric disorders. Journal of the American Academy of Child and Adolescent Psychiatry. Baltimore, v. 51, n. 9, p. 889-901, 2012. Disponível em: https://www.ncbi.nlm.nih.gov/pubmed/22917202 Acesso em: 09 jan. 2020.

BEYEA, Suzanne C.; NICOLL, Leslie H. Writing an integrative review. AORN Journal: the office voice of perioperative nursing. [s.i], v. 67, n. 4, p. 877-88, 1998. Disponível em: https://aornjournal.onlinelibrary.wiley.com/doi/abs/10.1016/S0001-2092\%2806\%29626537?sid=nlm\%3Apubmed. Acesso em: 26 dez. 2020.

CUERVO, Ángel Alberto Valdés; MARTÍNEZ, Ernesto Alonso Carlos; ACUÑA, Gisela Margarita Torres. Diferencias en la situación socioeconómica, clima y ajuste familiar de estudiantes con reportes de bullying y sin ellos. Psicología desde el Caribe. Barranquílla, v. 29, n. 3, p. 616-31, 2012. Disponível em: https://www.redalyc.org/pdf/213/21328599004.pdf. Acesso em: 10 jul. 2020. 
FARIA, Jeana Taize. O papel dos pais no desenvolvimento da resiliência em vítimas de bullying escolar. Cadernos Brasileiro de Saúde Mental. Florianópolis, v. 12, n. 3, p. 1-14, 2020.

Disponível em: https://periodicos.ufsc.br/index.php/cbsm/article/view/69671. Acesso em: 05 ago. 2020.

FARINA, Eleonora; BELACCHI, Carmem. The relationship between emotional competence and hostile/prosocial behavior in Albanian preschoolers: an exploratory study. School Psychology International. Miami, v. 35, n. 5, p. 475-84, 2014. Disponível em: https://journals.sagepub.com/doi/10.1177/0143034313511011. Acesso em: 10 jul. 2020.

FLEMING, Lila C.; JACOBSEN, Kathryn H. Bullying among middle-school students in low and middle income countries. Health Promotion International. Oxford, v. 25, n. 1, p. 73-84, 2010. Disponível em: https://doi.org/10.1093/heapro/dap046. Acesso em: 23 dez. 2020.

FREIRE, Alane Novais; AIRES, Januária Silva. A contribuição da psicologia escolar na prevenção e no enfrentamento do Bullying. Psicologia Escolar e Educacional, São Paulo, v. 16, n. 1, p. 55-60, 2012. Disponível em: https://doi.org/10.1590/S1413-85572012000100006 Acesso em: 30 dez. 2020.

GODLESKI, Stephanie A. et al. Peer victimization and peer rejection during early childhood. Journal of clinical child and adolescent psychology. London, v. 44, n. 3, p. 380-92, 2015. Disponível em: https://www.ncbi.nlm.nih.gov/pubmed/25133659. Acesso em: 09 jan. 2020.

HELGELAND, Anne; LUND, Ingrid. Children's voices on bullying in Kindergarten. Early Childhood Education Journal. [s.i], v. 45, n. 1, p. 133-41, 2017. Disponível em: https://link.springer.com/article/10.1007/s10643-016-0784-z. Acesso em: 09 jan. 2020.

IBEZIAKO, Patricia. et al. Bullying victimization in medically hospitalized patients with somatic symptom and related disorders: prevalence and associated factors. Hospital pediatrics. Elk Grove Village, v. 6, n. 5, p. 290-6, 2016. Disponível em: https://doi.org/10.1542 / hpeds.2015-0207 Acesso em: 10 ago. 2020.

JANSEN, Pauline W. et al. Teacher and peer reports of overweight and bullying among young primary school children. Pediatrics. Springfield, v. 134, n. 3, p. 473-80, 2014. Disponível em: https://www.ncbi.nlm.nih.gov/pubmed/25157018. Acesso em: 09 jan. 2020.

KENNEDY-BEHR, Ann; RODGER, Sylvia A.; MICKAN, Sharon. Aggressive interactions during free-play at preschool of children with and without developmental coordination disorder.

Research in developmental disabilities. New York, v. 34, n. 9, p. 2831-7, 2013. Disponível em: https://www.ncbi.nlm.nih.gov/pubmed/23796458. Acesso em: 09 jan. 2020.

KNOUS-WESTFALL, Heather M. et al. Parental intimate partner violence, parenting practices, and adolescent peer bullying: a prospective study. Journal of Child and Family Studies. South Carolina, v. 21, p. 754-66, 2012. Disponível em: https://ink.springer.com/article/10.1007/s10826011-9528-2. Acesso em: 05 jul. 2020. 
KONTAK, Julia C. H. et al. The relationship between bullying behaviours in childhood and physician-diagnosed internalizing disorders. Canadian Journal of Public Health. Otawa, v. 110, n. 4, p. 497-505, 2019. Disponível em: https://doi.org/10.17269/s41997-019-00179-3. Acesso em: 10 ago. 2020.

LEE, Seung-há; JU, Hyun-jung. Mothers' difficulties and expectations for intervention of bullying among young children in South Korea. International Journal of Environmental Research and Public Health. Basel, v. 16, n. 6, p. 924, 2019. Disponível em: http://doi.org/10.3390/ijerph16060924. Acesso em: 09 jan. 2020.

MARCOLINO, Emanuella de Castro. et al. Bullying: prevalência e fatores associados à vitimização e à agressão no cotidiano escolar. Texto \& Contexto - Enfermagem. Florianópolis, v. 27, n. 1, e5500016, 2018. Disponível em: https://www.scielo.br/pdf/tce/v27n1/0104-0707-tce27-01-e5500016.pdf. Acesso em: 05 jul. 2020.

MELLO, Flávia C. M. et al. A prática de bullying entre escolares brasileiros e fatores associados, pesquisa nacional de saúde do escolar 2015. Ciência \& Saúde Coletiva. Rio de Janeiro, v. 22, n. 9, p. 2939-48, 2017. Disponível em: https://doi.org/10.1590/1413-81232017229.12762017. Acesso em: 23 dez. 2020.

MENDES, Carla Silva. Prevenção da violência escolar: avaliação de um programa de intervenção. Revista da Escola de Enfermagem da USP. São Paulo, v. 45, n. 3, p. 581-8, 2011. Disponível em: https://www.scielo.br/scielo.php?script=sci_arttext\&pid=S008062342011000300005. Acesso em: 10 jul. 2020.

MENDES, Karina Dal Sasso; SILVEIRA, Renata Cristina de Campos Pereira; GALVÃO, Cristina Maria. Revisão integrativa: método de pesquisa para a incorporação de evidências na saúde e na enfermagem. Texto \& Contexto - Enfermagem. Florianópolis, v. 17, n. 4, p. 758-64, 2008. Disponível em: http://www.scielo.br/scielo.php?script=sci_arttext\&pid=S010407072008000400018. Acesso em: 09 jan. 2020.

MOHER, David. et al. Preferred reporting items for systematic reviews and meta-analyses: the PRISMA statement. Plos Medicine. San Francisco, v. 151, n. 4, p. 264-9, 2009. Disponível em: https://www.acpjournals.org/doi/10.7326/0003-4819-151-4-200908180-00135. Acesso em: 07 jul. 2020.

MOORE, Tim. et al. The first thousand days: an evidence paper. Victoria: Centre for Community Child Health, 2017.

NAVARRO, Jose-Blas. et al. Warning signs of preschool victimization using the strengths and difficulties questionnaire: prevalence and individual and family risk factors. Plos One. San Francisco, v. 14, n. 8, p. e0221580, 2019. Disponível em: http://doi.org/10.1371/journal.pone.0221580. Acesso em: 10 jan. 2020.

NATIONAL CENTER FOR EDUCATION STATISTICS. Student reports of bullying: results from the 2015 school crime supplement to the national crime victimization survey. Departament of Education: Washington, 2016. 
OKSENDAL, Elise. et al. Peer-victimization of young children with developmental and behavioral difficulties - a population-based study. Journal of Pediatric Psychology. Washington, v. 44, n. 5, p. 589-600, 2019. Disponível em: http://doi.org/10.1093/jpepsy/jsy112. Acesso em: 10 jan. 2020.

OLIVEIRA, Wanderlei Abadio. et al. Family interactions and the involvement of adolescents in bullying situations from a bioecological perspective. Estudos em Psicologia. Campinas, v. 37, e180094, 2020. Disponível em: https://doi.org/10.1590/1982-0275202037e180094. Acesso em: 10 ago. 2020.

OLIVEIRA, Wanderlei Abadio. et al. Revisão sistemática sobre bullying e família: uma análise a partir dos sistemas bioecológicos. Revista de Salud Pública. Bogotá, v. 20, n. 3, p. 396-403, 2018. Disponível em: https://doi.org/10.15446/rsap.V20n3.47748. Acesso em: 12 ago. 2020.

OLSON, Sheryl. et al. Individual differences in the development of early peer aggression: Integrating contributions of self-regulation, theory of mind, and parenting. Development and Psychopathology. New York, v. 23, n. 1, p. 253-66, 2011. Disponível em: https://www.ncbi.nlm.nih.gov/pubmed/21262052. Acesso em: 09 jan. 2020.

OLWEUS, Dan. School bullying: development and some important challenges. Annual review of clinical psychology. Palo Alto, v. 9, n. 1, p. 751-780, 2013. Disponível em: https://doi.org/10.1146/annurev-clinpsy-050212-185516. Acesso em: 30 dez. 2020.

RAJENDRAN, Khushmand; KRUSZEWSKI, Edyta; HALPERIN, Jeffrey M. Parenting style influences bullying: A longitudinal study comparing children with and without behavioural problems. Journal of Child Psychology and Psychiatry, and Allied Disciplines. New York, v. 57, n. 2, p. 188-95, 2016. Disponível em: https://www.ncbi.nlm.nih.gov/pmc/articles/PMC4670820. Acesso em: 09 jan. 2020.

SANTOS, Cristiane Flores; LOPES, Fernando José. O bullying na escola em crianças com hemofilia. Revista de Pós-graduação Multidisciplinar. São Paulo, v. 1, n. 1, p. 353-60, 2017. Disponível em: http://fics.edu.br/index.php/rpgm/article/view/524/572. Acesso em: 08 jul. 2020.

SCHULTZ Naiane Carvalho Wendt, et al. A compreensão sistêmica do bullying. Psicologia em Estudo, Maringá, v. 17, n. 2, p. 247-54, 2012. Disponível em: http://dx.doi.org/10.1590/S141373722012000200008. Acesso em: 30 dez. 2020.

SHETGIRI, Rashmi; LIN, Hua Lin; FLORES, Glenn. Trends in risk and protective factors for child bullying perpetration in the United States. Child Psychiatry and Human Development. New York, v. 44, n. 1, p. 89-104, 2013. Disponível em: https://pubmed.ncbi.nlm.nih.gov/22661150. Acesso em: 05 jul. 2020.

SHONKOFF, Jack P. Protecting brains, nor simply stimulating minds. Science. Washington, v. 333, n. 6045, p. 982-3, 2011. Disponível em: https://www.ncbi.nlm.nih.gov/pubmed/21852492. Acesso em: 09 jan. 2020.

SHONKOFF, Jack P.; BOYCE, Thomas; McEWEN, Bruce S. Neuroscience, molecular biology, and the childhood roots of health disparities: building a new framework for health promotion and disease prevention. JAMA. Chicago, v. 301, n. 21, p. 2252-9, 2009. Disponível em: https://www.ncbi.nlm.nih.gov/pubmed/19491187. Acesso em: 09 jan. 2020. 
SHONKOFF, Jack P.; GARNER, Andrew S. The lifelong effects of early childhood adversity and toxic stress. Pediatrics. Springfield, v. 129, n. 1, e232-46, 2012. Disponível em: https://uww.ncbi.nlm.nih.gov/pubmed/22201156. Acesso em: 09 jan. 2020.

SILVA, Anne Heracléia Brito; ARAÚJO, Laiane de Sousa. Bullying: uma expressão da questão social. Serviço Social \& Realidade. Franca, v. 24, n. 2, p. 127-42, 2015. Disponível em: https://periodicos.franca.unesp.br/index.php/SSR/article/view/2738/2341. Acesso em: 08 jul. 2020.

SILVA, Fernanda; DASCANIO, Denise; VALLE, Tânia Gracy Martins. O fenômeno bullying: diferenças entre meninos e meninas. Revista Reflexão e Ação. Santa Cruz do Sul, v. 24, n. 1, p. 26-46, 2016. Disponível em: https://online.unisc.br/seer/index.php/reflex/article/view/7014/pdf. Acesso em: 05 jul. 2020.

SILVA, Jorge Luiz. et al. Intervention in social skills and bullying. Revista Brasileira de Enfermagem. Brasília, v. 71, n. 3, p. 1085-91, 2018. Disponível em: https://doi.org/10.1590/00347167-2017-0151. Acesso em: 17 ago. 2020.

SILVA, Jorge Luiz. et al. Revisão sistemática da literatura sobre intervenções antibullying em escolas. Ciência \& Saúde Coletiva, Rio de Janeiro, v. 22, n. 7, p. 2329-40, 2017. Disponível em: http://dx.doi.org/10.1590/1413-81232017227.16242015 Acesso em: 30 dez. 2020.

SON, Esther; PARISH, Susan L.; PETERSON, Andrew. National prevalence of peer victimization among young children with disabilities in the United States. Children and Youth Services Review. [s.i], v. 34, n. 8, p. 1540-5, 2012. Disponível em: https://www.sciencedirect.com/science/article/pii/S0190740912001727\#aep-keywords-id11. Acesso em: 09 jan. 2020.

THOMAS, Hannah J. et al. Prevalence and correlates of bullying victimization and perpetration in a nationally representative sample of Australian youth. The Australian and New Zealand journal of psychiatry. London, v. 51, n. 9, p. 909-20, 2017. Disponível em: https://doi.org/10.1177/0004867417707819. Acesso em: 17 ago. 2020.

ZEQUINÃO, Marcela Almeida. et al. Bullying escolar: um fenômeno multifacetado. Educação e Pesquisa. São Paulo, v. 42, n. 1, p. 181-98, 2016. Disponível em:

https://www.scielo.br/pdf/ep/v42n1/1517-9702-ep-42-1-0181.pdf. Acesso em: 10 jul. 2020.

ZYCH, Izabela. et al. Springer briefs in psychology. Protecting children against bullying and its consequences. New York, USA: Springer Science + Business Media, 2017. Disponível em: http://doi.org/10.1007/978-3-319-53028-4. Acesso em: 23 dez. 2020.



This work is licensed under a Creative Commons Attribution-NonCommercial 4.0 International (CC BY-NC 4.0) 